\title{
A Novel Direct Technique for Fabrication of Provisional Fixed Partial Denture
}

\author{
Ivan Chakalov ${ }^{1}$, Pavlina Ivanova ${ }^{2}$ \\ 1. Department of Prosthetic Dentistry, Faculty of Dental \\ Medicine, Medical University - Sofia, Bulgaria \\ 2. Dentist with a Private Practice - Sofia, Bulgaria
}

\begin{abstract}
The present article introduces a new technique for chairside provisional fixed partial dentures fabrication. The described direct technique is suitable for a fast and relatively easy fabrication of provisional single crowns or small bridges. It does not necessitate any preliminary laboratory stages (such as wax-up e.g.) but rather tends to adapt the teeth morphology of a selected model to the specific clinical case.
\end{abstract}

Key words: Provisional, interim, temporary, FPD, chairside, shell

\section{Background}

Various techniques have been described in literature for the fabrication of provisional fixed partial dentures (FPD). The most commonly used classification divides the pFPD techniques into direct, direct-indirect and indirect (1). The indirect allow a better visibility and understanding of the clinical case challenges for both dentist and dental technician and are recommended for complex cases involving significant changes in terms of teeth shape and position. However the preliminary stages required and therefore the additional laboratory cost, as well as the problems regarding the accurate information transmission from the model to the mouthmake direct techniques preferable method for pFPD fabrication (2). Direct techniques are further subdivided by some authors into shells, matrices and direct syringing (2). Another existing shell technique is the so-called "alginate shell". A brush is successively dipped into monomer and resin powder. The resultant slurry formed on the brush is than applied as a thin layer on the internal surface of an alginate impression from the preliminarily made wax-up.

\section{Technique}

The preliminary stage of the provisional FPD fabrication starts with the fabrication of silicon molds. Different sort of full arch models might be selected as patterns for the molds - study models from real patients made of dental stone or resin, presenting good anatomical and morphological characteristics, 
prefabricated anatomy models (sold by companies such as BREDENT), or preclinical student dental anatomy models (such as P.K.Thomas e.g.).A sectorial silicon matrix made of polyvinyl siloxane impression material (A-type of silicone) is made from the selected model. A putty consistency of polymethylsiloxane (C-type of silicone) is then prepared and pressed within the matrix forming a positive replica of the model. The two silicones will not adhere to each other because of a chemical incompatibility and are easily disassembled thus forming a negative mold and positive shell for pressing resin inbetween.

The clinical stage of the pFPD fabrication begins with the preparation of the teeth (Fig.1). The dental assistant prepares the self-curing resin which might be of any known type of provisional FPD material. The resin is then pressed between the two silicone components forming a thin resin shell (Fig.2) which is retrieved once it reaches the rubber-like stage of polymerization. The so-formed shell reproduces faithfully the anatomical characteristics of the initially selected anatomical model (Fig.3, 4), but at this stage is very easily deformable. A thin layer of a light-curing bonding agent is then applied on the surface of the shell, positioned in the mouth. The patient is asked to close in a maximum intercuspation (MIP) position and thus the adhesive is being light-cured. The shell is now adapted to the occlusion of the patient and possesses some rigidity. It takes one or two relinings to achieve the final margin adaptation, contour and the appropriate contacts with the adjacent teeth and antagonists. The thickness of the provisional FPD is checked in order to confirm that an adequate occlusal and axial clearance for the future definitive crown is been provided with the preparation. If any adjustments are needed, another relining would be necessary. The provisional FPD is then polished and characterized. Staining with white and brown light-curing colorants might be used to emphasize the anatomy and make the crown harmonize with the adjacent teeth. The provisional FPD might be fixed using any type of provisional cement (Fig. 5).

Fig. 1. Preparation of the tooth

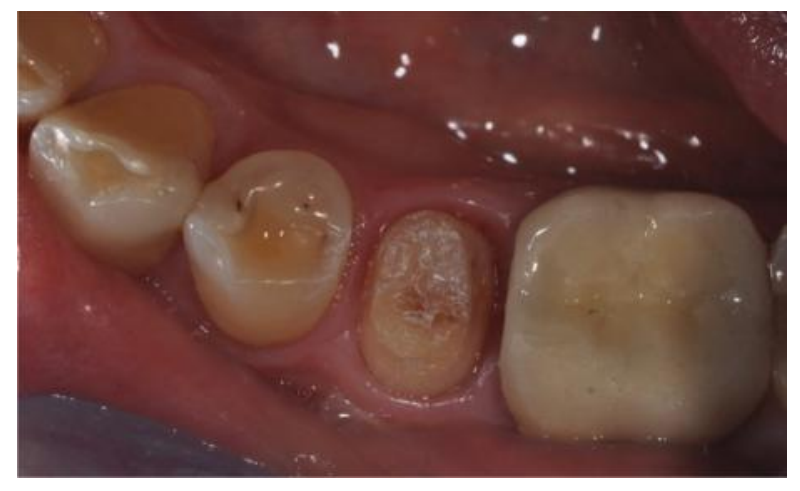


Fig. 2. Resin pressed between the two silicone components

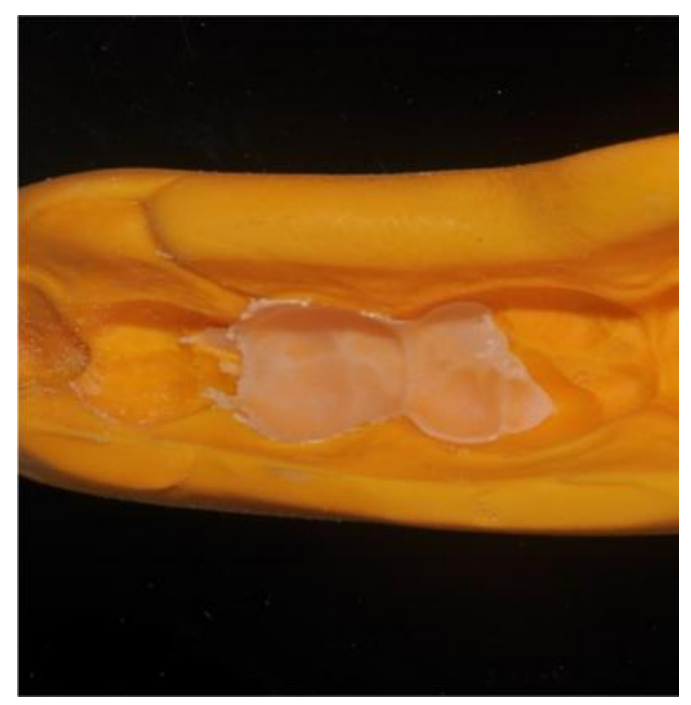

Fig. 3. A thin resin shell with uniform thickness

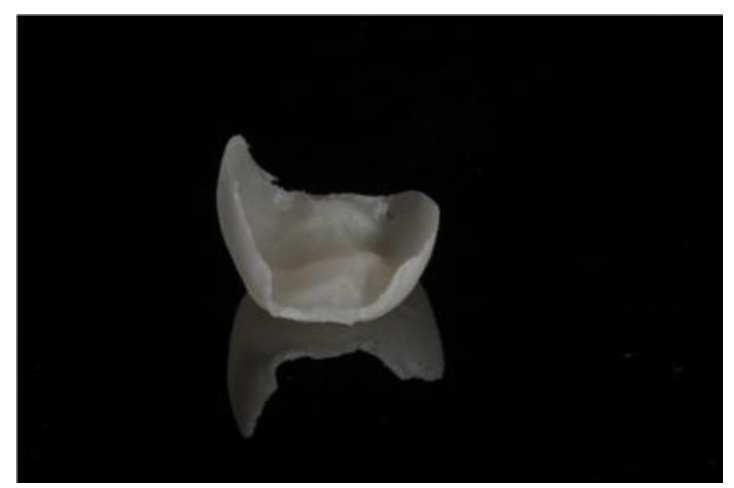

Fig. 4. All morphological characteristics are accurately reproduced

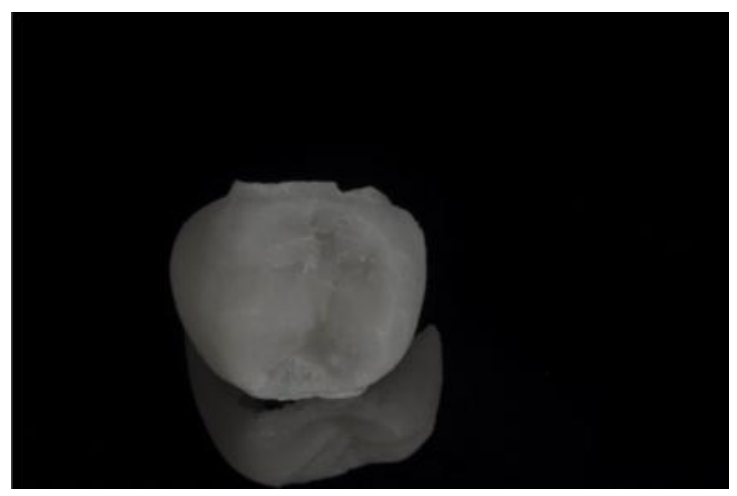


Fig.5 Cemented provisional crown.

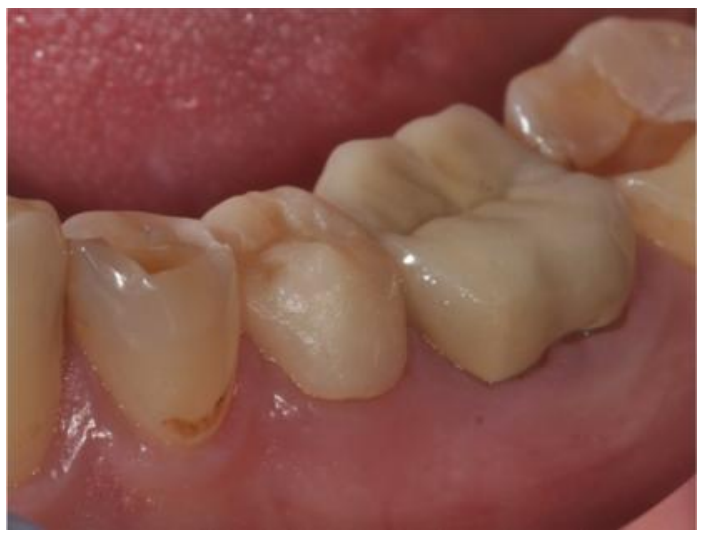

The overall time for provisional fabrication in this case did not exceed 15 minutes. The most time consuming stages which are anatomical details reproduction and occlusion are significantly reduced because of the technique.

Fig. 6. Comparison between provisional and final crown

Fig.7. Finished case

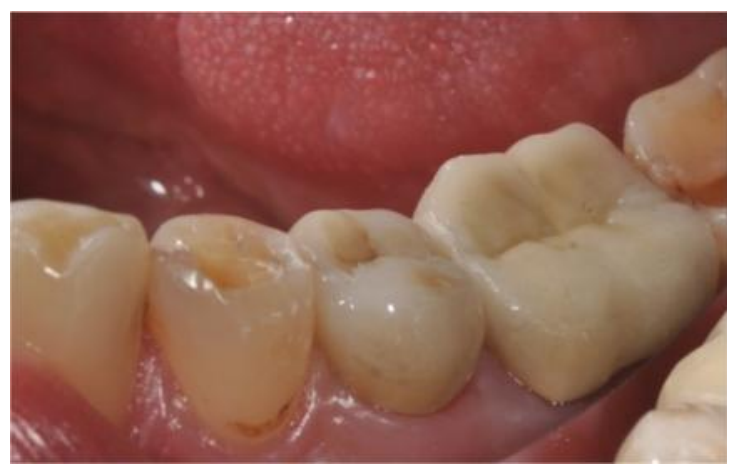




\section{Discussion}

The technique described in this article belongs to the direct acrylic shell methods. Rosenstiel (3) describes many techniques for interim FPD fabrication which he classifies according to the method of shaping the external and internal surface form. Within the shell techniques described in the literature a prefabricated patterns might be used, made out of cellulose acetate or polycarbonate, which need one or more relinings in order to fit properly the prepared teeth. Occlusal adjustments with these techniques might be sometimes excessively time-consuming. The advantage of the presented method is the ability to customize a preliminary selected crown morphology as well as rapidly fabricate a regular thickness shell with the desired morphology. The external staining of provisional FPD's enhances the esthetic appearance and reproduces the natural look of the teeth.

From the dental materials point of view it is advisable to have provisional FPD's produced out of heatcuring than in self-curing resin because of the lower residual monomer content. The teeth on the model used as reference should be dimensionally similar to the ones in the clinical situation. Thus the need to use several template models with different dimensional characteristics

\section{Conclusion}

The described technique is a relatively easy and rapid way of chairside fabrication of pFPDs. Same technique might be applied for making direct overlays, single crowns and several-unit bridges. The obtained shape preserves in most cases the beautiful morphological characteristics of the initial anatomical model. Pictures of the finished and stained crown might be sent to the laboratory during the color selection phase as an additional motivational tool for the dental technicians. Furthermore the thickness of the finished and occlusally adjusted pFPD is the perfect indicator to self-control the occlusal clearance of the preparation.

\section{References}

1. ShillingburgH.T, Hobo S, Whitsett LD, et al. Fundamentals of Fixed Prosthodontics Publisher:QuintessencePublishing Co 1997 book.

2. Wassell W, George St, Ingledew $P$, et al. Crownsandotherextra-coronalrestorations: ProvisionalrestorationsBritishDentalJournal 2002;192:619-630

3. Rosenstiel F, Land F, Fujimoto J. ContemporaryFixedProsthodonticsPublisher: Mosby, Inc.2006 book.

\section{Corresponding author}

Ivan Chakalov

Prosthetic Dentistry

Faculty of Dental Medicine,

Medical University - Sofia; Georgi Sofiyski blvd., 1431 Sofia, Bulgaria; +359 888937264 